\title{
LA FLORA MEDICINAL ANDINA EN LAS PREPARACIONES tRADICIONALES DE LA COMUNIDAD de Ocumazo (Pueblo Omaguaca), Jujuy, Argentina
}

\author{
The Andean medicinal FLORA In the tRAditionAL PREPARATIONS OF THE \\ community of Ocumazo (Pueblo Omaguaca), Jujuy, Argentina
}

\author{
Gisella L. Tortoni ${ }^{\star 1,2}\left[\mathbb{C}\right.$, Bárbara Arias Toledo ${ }^{3}\left[\right.$ y Nilda D. Vignale ${ }^{1,2}(\mathbb{C}$
}

\begin{abstract}
1. Instituto de Ecorregiones Andinas - INECOA (CONICET UNJu, Argentina).

2. Grupo de Etnobiología y Micrografía Aplicada (GEMA)

3. Instituto Multidisciplinario de Biología Vegetal - IMBIV (CONICET - UNC), Argentina.
\end{abstract}

*gisellatortoni@gmail.com

\section{Citar este artículo}

TORTONI, G. L., B. ARIAS TOLEDO \& N. D. VIGNALE. 2021. La flora medicinal andina en las preparaciones tradicionales de la comunidad de Ocumazo (Pueblo Omaguaca), Jujuy, Argentina. Bol. Soc. Argent. Bot. 56: 403-417.

DOI: https://doi. org/10.31055/1851.2372.v56. n3.32953

Recibido: 6 May 2021

Aceptado: 4 Ago 2021

Publicado en línea: 8 Sep 2021

Publicado impreso: 30 Set 2021

Editora: Norma Hilgert (iD

ISSN versión impresa 0373-580X

ISSN versión on-line 1851-2372

\section{SUMmaRY}

Introduction: Traditional knowledge about medicinal plants is part of the health system of indigenous communities. The use of multiple combined species is frequent in the Argentine Northwest, but studies focused on the subject are scarce. This study describes and contextualizes the uses of these preparations.

M\&M: We applied participant observation techniques in the indigenous community of Ocumazo, Humahuaca, Jujuy, Argentina, with its prior informed consent. Open interviews were undertaken with key collaborators and other adults, through sampling by networks and by opportunity. We investigated the elaboration of recipes and how people used them. Further, we analyzed the administration and the context of application. The data were analyzed with qualitative techniques.

Results: 12 combinations of 26 plant species were surveyed (69\% native, $23 \%$ cultivated, $9 \%$ adventitious), including animal products. In daily life, medicinal preparations are used for respiratory, gastrointestinal, bone, and musculoskeletal diseases, among others. In the context of ritual celebration, the beverages take on symbolic significance.

Conclusions: The inhabitants of Ocumazo articulate precise knowledge about the ways to combine the species and to administrate the formulas. In a ritual context, the selection of species and the elaboration of recipes differ from daily use. The community has its own knowledge about the distribution of species according to warm or cold qualities prevailing notions of Hippocratic humoral medicine.

\section{KEY WORDS}

Ethnobotany, ethnomedicine, indigenous peoples, medicinal recipes, traditional botanical knowledge.

\section{Resumen}

Introducción: El conocimiento tradicional sobre plantas medicinales forma parte del sistema de salud de comunidades indígenas. La utilización de especies combinadas es habitual en el Noroeste argentino, más los estudios al respecto son escasos. Se describen y contextualizan los usos de dichos preparados.

M\&M: Con el consentimiento previo informado de la comunidad indígena de Ocumazo, Humahuaca, Jujuy, Argentina se aplicaron técnicas de observación participante y entrevistas abiertas a colaboradores clave y otros adultos, mediante muestreo por redes y por oportunidad. Los datos sobre la elaboración de las preparaciones, usos, administración y contexto de aplicación se analizaron con técnicas cualitativas.

Resultados: Se relevaron 12 combinaciones de 26 especies vegetales (69\% nativas, $23 \%$ cultivadas, $9 \%$ adventicias), incluyendo productos animales. En el marco de la vida cotidiana se utilizan para tratar afecciones óseas y músculo-articulares, gastrointestinales, templarias, entre otras. En contexto ritual los preparados cobran significación simbólica.

Conclusiones: La comunidad de Ocumazo articula conocimientos precisos sobre la combinación de especies y la administración de las fórmulas. En contexto ritual configura diferentes pautas de selección, elaboración y consumo respecto al uso cotidiano. Existen conocimientos propios acerca de la distribución de las especies según cualidades cálidas o frías, prevaleciendo nociones de la medicina humoral hipocrática.

\section{Palabras clave}

Conocimiento botánico tradicional, etnobotánica, etnomedicina, preparaciones medicinales, pueblos originarios. 


\section{INTRODUCCIÓN}

Las prácticas terapéuticas y los conocimientos medicinales transmitidos oralmente a través de varias generaciones, constituyen la base de los sistemas de salud de las comunidades originarias (OMS, 2003). En estos pueblos se evidencia una preferencia por la auto atención como primera estrategia para actuar sobre la dolencia propia y de los cercanos (Drovetta, 2009; Martínez, 2011). La medicina tradicional es definida como «prácticas, enfoques, conocimientos y creencias sanitarias diversas que incorporan medicinas basadas en plantas, animales y minerales ... aplicados de forma individual o en combinación para mantener el bienestar, además de tratar, diagnosticar y prevenir las enfermedades»(OMS, 2003). De este modo, millones de personas en el mundo recurren a hierbas $\mathrm{y}$ tratamientos tradicionales como principal fuente de atención médica, en muchos casos la única disponible (OMS, 2013).

El conocimiento botánico tradicional (CBT) es definido como un cuerpo acumulativo de conocimientos, prácticas y creencias acerca de las relaciones entre las sociedades y los componentes vegetales de su entorno (Berkes, 1993). Estudios realizados en el noroeste argentino(NOA) evidencian que el CBT guía los procesos de mantenimiento y restauración de la salud, como también la gestión de los recursos naturales comunitarios (Pochettino \& Lema, 2008; Vignale \& Pochettino, 2009). Particularmente, los pueblos indígenas de la región andina se valen de diferentes estrategias de atención y cuidado de la salud, imbricadas en múltiples tramas rituales, religiosas y cotidianas. En estos diversos contextos, la utilización de especies vegetales combinadas y de sustancias animales ha sido informada previamente por diferentes autores (Hilgert \& Gil, 2006; Crivos et al. 2009; Idoyaga Molina \& Sarudiansky, 2011). Adicionalmente, varios estudios se han ocupado de analizar la prevalencia de la teoría calor y frío en los sistemas etnomédicos de Latinoamérica y Argentina (Foster, 1953; Currier, 1966; Idoyaga Molina, 1999a; Scarpa, 2002, 2004; Bianchetti, 2016). Esta teoría se basa en la idea de la existencia de cierto equilibrio en la temperatura del cuerpo, el cual puede alterarse debido a la exposición excesiva al calor y al frío, así como a la sequedad y la humedad (Hilgert, 2001; Hilgert \& Gil 2006, 2007; Martínez, 2008). El desbalance generado en la condición corporal causaría afecciones cálidas y frías, tratadas -en general- mediante la administración de elementos con características térmicas complementarias a la enfermedad (Queiroz, 1984).

Una problemática planteada, décadas anteriores, en la investigación sobre usos de las plantas es la ausencia de discusiones vinculadas al contexto cultural y ritual de las enfermedades y las prácticas terapéuticas (Alexiades, 1995). Algunos estudios etnobotánicos en el NOA han generado valiosas contribuciones a la superación del asunto (Martínez \& Pochettino, 2004; Hilgert, 2004, 2009). Con todo, el estudio de las preparaciones medicinales y su contexto de uso en la región ha sido -en generaldesarrollado de forma secundaria respecto de otras temáticas predominantes. En este marco, el presente artículo se propuso describir y contextualizar los usos de preparados medicinales por parte de la comunidad de Ocumazo, Departamento de Humahuaca, Jujuy, Argentina.

\section{Materiales y Métodos}

\section{Área de estudio}

Ocumazo se encuentra localizada en la Quebrada de Humahuaca, a $18 \mathrm{~km}$ al sudeste de la ciudad de Humahuaca, Departamento homónimo, Provincia de Jujuy, Argentina (Fig. 1) a una altitud de 3050 metros sobre el nivel del mar (msnm) (S23 ${ }^{\circ} 14 ' 13,0^{\prime \prime}$ W $\left.065^{\circ} 16^{\prime} 41,5^{\prime \prime}\right)$. Su temperatura media anual es de $11.1{ }^{\circ} \mathrm{C}$ y la precipitación aproximada de $202 \mathrm{~mm}$, y pertenece a la provincia fitogeográfica prepuneña (Cabrera, 1971). La vegetación dominante es la estepa arbustiva xerófila, además están presentes cardonales, bosquecillos enanos y cojines de bromeliáceas. Entre las especies dominantes se identifican Adesmia inflexa Griseb., Aphyllocladus spartioides Wedd., Arquita trichocarpa (Griseb.) Gagnon G.P.Lewis \& C.E.Hughes, Baccharis boliviensis (Wedd.) Cabrera, Bougainvillea spinosa (Cav.) Heimerl, Bulnesia schickendantzii Hieron. ex Griseb., Chuquiraga erinacea D. Don, Junellia juniperina (Lag.) Moldenke, Justicia floribunda (C. Koch) Wassh., Krameria lappacea (Dombey) Burdet \& B.B. Simpson, Lycium tenuispinosum Miers, $L$. ciliatum Schltdl., Parkinsonia andicola (Griseb.) Varjão \& Mansano, Pentaphorus glutinosus D. Don, Proustia cuneifolia D. Don, Senna crassiramea (Benth.) H.S. Irwin \& Barneby, Zuccagnia punctata 


\section{G. L. Tortoni et al. - Preparaciones medicinales de Ocumazo}
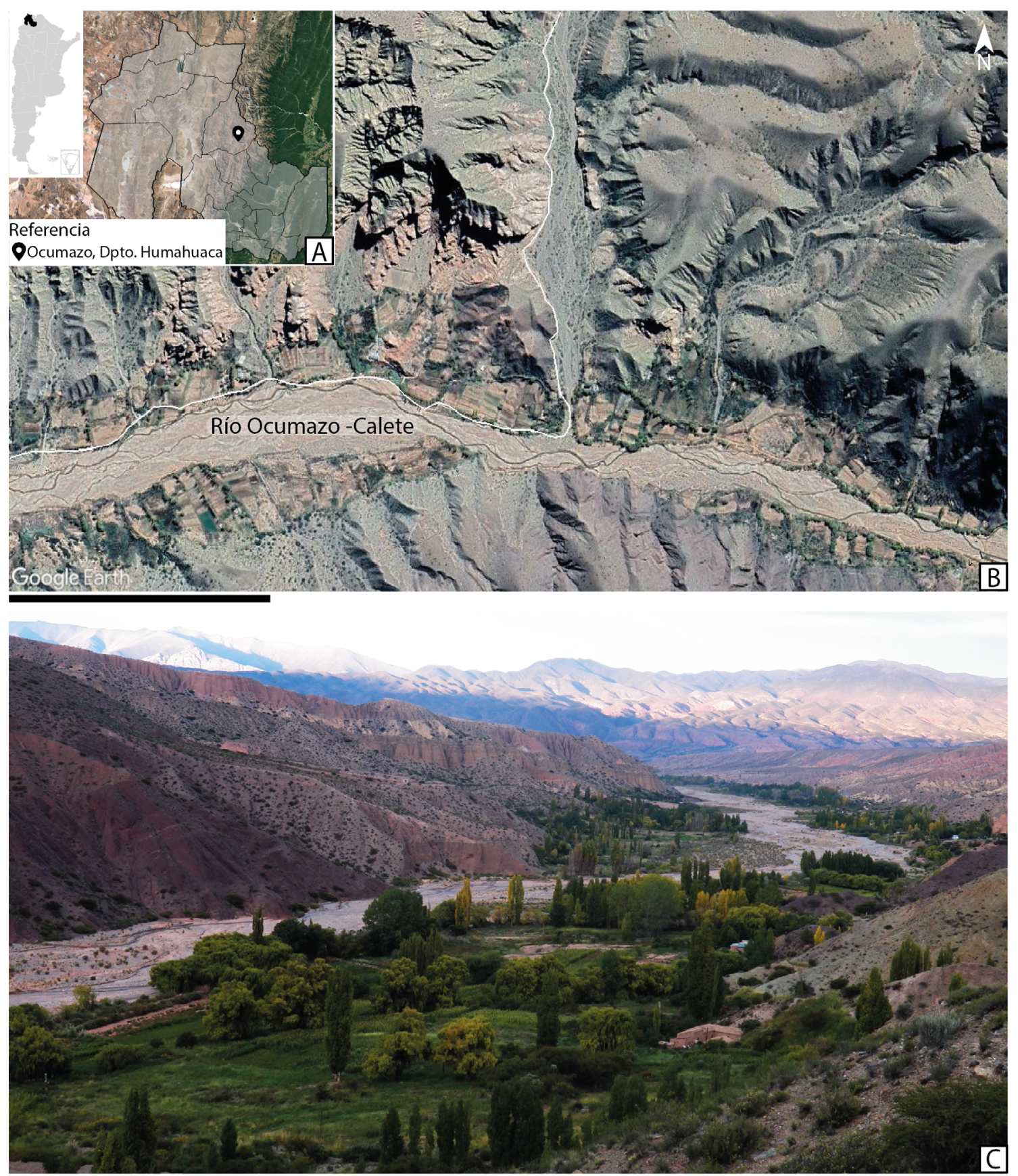

Fig. 1. Localización del sitio de estudio. A: Mapa de Argentina y de la Provincia de Jujuy. B: Imagen satelital de Ocumazo. C: Fotografía del paisaje de Ocumazo. Abreviaturas= Dpto: departamento. Escala= B: 800m

Cav., y cardonales de Trichocereus atacamensis (Phil.) W.T. Marshall \& T.M. Bock y T. terschecki. (J. Parm. ex Pfeiff.) Britton \& Rose. Comunidades de Prosopis ferox Griseb. se encuentran en el fondo de las quebradas secas y matorrales de Baccharis salicifolia (Ruiz \& Pav.) Pers. y Schinus areira L. en las orillas de los ríos (Aagesen et al. 2009; Cabrera, 1971, 1973, 1976). 


\section{Contexto sociocultural}

El acceso a Ocumazo puede realizarse a través de dos caminos vehiculares de tierra y ripio, y por un sendero peatonal desde la ciudad de Humahuaca (Fontes, 2017). El servicio de salud más accesible es el centro de atención primaria (CAP), ubicado a metros del centro vecinal y la iglesia, mientras que el Hospital General Manuel Belgrano de Humahuaca es el más cercano.

La comunidad se compone por treinta y seis familias originarias del pueblo Omaguaca (García Moritán \& Cruz, 2011), siendo setenta personas habitantes del lugar según lo registrado en el censo comunal. Residen de forma permanente tres a cuatro familias, el resto de la población ha migrado a diferentes localidades urbanas como Humahuaca, San Salvador de Jujuy, Palpalá y Buenos Aires, entre otras, en busca de oportunidades laborales y educativas principalmente. En general, visitan frecuentemente la comunidad para asistir a la asamblea comunitaria mensual y para instalarse temporalmente en momentos específicos del año como la época de siembra, de cosecha, de poda de frutales y también durante las celebraciones festivas y religiosas (Muñoz, 2014). Varios residentes de Humahuaca, nativos de Ocumazo, incluso se trasladan hacia allí algunos días de la semana dependiendo del trabajo que deban realizar en el campo y el régimen escolar de los hijos o nietos. De esta forma las familias, aun habiendo migrado, mantienen un sentido de pertenencia a la comunidad explicitada en contactos previos al inicio de esta investigación.

La actividad productiva principal en el territorio es la frutihortícola destinada al autoconsumo, como también a los mercados locales para su comercialización. Se realiza en parcelas ubicadas en los márgenes del río Calete que atraviesa al poblado. Tal producción incluye frutales como "manzanas" (Malus domestica Borkh) y "duraznos" (Prunus pérsica (L.) Batsch) entre otras (Villalba \& Lambaré, 2019). A partir de estas materias primas se elaboran mermeladas y sidra, entre otros derivados.

\section{Metodología y análisis de datos}

El relevamiento de datos se realizó, en primer lugar, aplicando técnicas de observación participante, según propone Guber (2001), durante ocho instancias que incluyeron la presencia en asambleas comunitarias mensuales, en ferias y festividades. Específicamente en la "Feria y festival de la manzana y sus derivados" -marzo 2018, Ocumazo, organizada por la comunidad-, en la challada de la Pachamama - 2018, Ocumazo-, en la "12ava Feria de las semillas nativas y criollas" julio 2018, Humahuaca, y en el "1er Cambalache" -agosto 2018, Aparzo-.

Adicionalmente, se realizaron entrevistas abiertas y semiestructuradas a siete mujeres y dos varones en un rango etario de cincuenta a ochenta años. El cuestionario estuvo orientado a la indagación del CBT medicinal, incluyendo nombres populares, usos, formas de preparación, administración, abastecimiento de los recursos vegetales y prácticas curativas que los incluyen. Los colaboradores entrevistados fueron seleccionados a través de muestras por redes ("bola de nieve") para la búsqueda de colaboradores especializados, y por oportunidad en el caso de personas que se encontraban disponibles e interesadas en dialogar al momento de la visita (Hernández Sampieri et al., 2014). Se contó con el consentimiento previo informado, comunitario escrito e individual oral, de acuerdo a lo establecido por el Código de Ética de la Sociedad Internacional de Etnobiología (ISE, 2006).

Para colectar las especies mencionadas se efectuaron recorridas a campo, siempre que fue posible, en compañía de los colaboradores. El permiso de colección fue otorgado por la Secretaria de Biodiversidad, Ministerio de Ambiente de la Provincia de Jujuy (Expediente 1103- $\mathrm{N}^{\circ}$ 091D/2019).

Los usos medicinales de cada preparado se designaron en nomenclatura propia de la medicina científica, a través del agrupamiento de expresiones $\mathrm{y}$ términos populares diversos en afecciones categorizadas y adaptadas de Hilgert (2009). El material botánico colectado fue herborizado e identificado en gabinete, mediante observación en lupa y consulta bibliográfica. Los ejemplares de referencia se depositarán en el herbario del Museo Botánico de la Universidad Nacional de Córdoba (CORD), en el presente trabajo se indican bajo el número de colección personal de la primera la autora (GLT). Los materiales fragmentados fueron depositados en el muestrario del Grupo de Etnobiología y Micrografía Aplicada (M- GEMA).

Para actualizar los nombres científicos y estatus de las especies (nativa, cultivada o adventicia) se 


\section{G. L. Tortoni et al. - Preparaciones medicinales de Ocumazo}

emplearon las bases de datos Flora del Cono SurFlora Argentina (IBODA, 2019) y el Catálogo de las Plantas Vasculares del Cono Sur (Zuloaga et al., 2017) versión online. Se calcularon los porcentajes de especies para cada estatus respecto del total de especies citadas.

\section{Resultados}

Se relevaron doce combinaciones de veintiséis taxones, correspondientes a dieciocho familias botánicas. La lista de familias, especies, nombres locales y estatus botánico se indica en la Tabla 1. Del total de especies, $69 \%$ son nativas, $23 \%$ cultivadas y $8 \%$ adventicias.

Asimismo, se recopilaron datos sobre preparados con plantas que incorporan productos de origen animal. Dado que solo las preparaciones yerbeado $\mathrm{y}$ alcohol alcanforado fueron mencionadas con un nombre propio, al resto de los combinados se les asignó arbitrariamente un número determinado. Esta información, los usos, nombres locales y forma de administración se detallan en la Tabla 2.

En cuanto al abastecimiento de los materiales vegetales empleados para elaborar las preparaciones, los colaboradores mencionaron que depende, principalmente, de la disponibilidad del recurso en el lugar según la época del año. Precisamente, en temporadas de verano y otoño realizan recolección directa para utilizar la planta en estado fresco. En cambio, en períodos de sequía recurren al uso de hierbas colectadas durante la época en la que aún "está verde". Para este fin, el procedimiento habitual consiste en colectar, lavar, disecar y, posteriormente, almacenarlas en la vivienda. La Semana Santa - conmemoración cristiana de Pascua, de fecha variable de acuerdo al calendario litúrgico, entre los meses de marzo y abril- es considerada el momento ideal para la recolección ya que se afirma que en este periodo "están bendecidos los yuyos". En caso de no disponer del recurso usualmente acuden al apoyo de algún familiar/ vecino o lo compran en ferias locales. Sin embargo, los hábitos de vida de las plantas que crecen exclusivamente a más de $3000 \mathrm{msnm}$ representan un obstáculo para las personas mayores, dada la imposibilidad de acceder a esos espacios por sus condiciones de salud, problemática que muchas veces no pueden salvar debido a la ausencia de jóvenes en el lugar que puedan buscarlos por ellos.
Respecto a los usos informados para cada preparación se observó que las afecciones óseas músculo-articulares (OA) reportaron una mayor cantidad de fórmulas medicinales. En segundo lugar, se ubican las gastrointestinales (GI), seguidas de las templarias (T) asociadas al enfriamiento del cuerpo. Para las OA se mencionaron estrategias que van desde el uso de infusiones y baños, hasta fricciones con "pomadas" confeccionadas a partir de recursos vegetales combinados con alcohol y derivados animales.

Cualidades templarias de las plantas y su distribución

En cuanto a la identificación de las especies cálidas y frías, una de las colaboradoras clave mencionó que las plantas no poseen rasgos particulares que permitan clasificarlas y, por lo tanto, la asignación del carácter cálido o fresco se aprendería de memoria a través de la transmisión oral del conocimiento. Sin embargo, otra especialista argumentó que la distribución de las especies está asociada con la altura, afirmando que: "las (plantas) que crecen en la altura son para curar el frío y abajo para curar si se calorean". Los datos analizados en el presente estudio apuntan al uso de baños para tratar principalmente el "resfrio" $o$ "refrio", dolencia adquirida en terrenos de elevación mayor a $3200 \mathrm{msnm}$ aproximadamente, para la cual se indican síntomas como "dolor de cuerpo, de huesos, de columna... debilita a los huesos porque es más frío allá " haciendo referencia a los cerros, donde las temperaturas son más bajas respecto al valle en el que se asienta la comunidad.

\section{Preparaciones de uso externo}

Las fórmulas empleadas de manera externa incluyen baños (Tabla 2, preparados $\mathrm{N}^{\circ} 1$ a 3 ) con infusiones calientes utilizados para el tratamiento de afecciones templarias, con el fin de restablecer el equilibrio cálido-frío.

Las fórmulas administradas a través de fricciones sobre la piel son utilizadas exclusivamente para aliviar dolores musculares, óseos y articulares, y requieren de procesos de elaboración más complejos. Para el caso del llamado "alcohol alcanforado" (Tabla 2, $\mathrm{N}^{\circ} 5$ ) esto implica la adición de alcohol al material vegetal y de una pequeña parte de extracto de alcanfor, comprado en locales comerciales de ciudades cercanas. Más aún, la mixtura $\mathrm{N}^{\circ} 6$ (Tabla 2) requiere de la elaboración 
Bol. Soc. Argent. Bot. 56 (3) 2021

Tabla 1. Especies mencionadas para la elaboración de preparados medicinales, ordenadas alfabéticamente según familia, indicando nombre popular y status botánico.

\begin{tabular}{|c|c|c|c|}
\hline Familia/ nombre científico & $\begin{array}{l}\text { Nombre } \\
\text { popular }\end{array}$ & Voucher, fecha y lugar & Estatus \\
\hline \multicolumn{4}{|l|}{ Amaryllidaceae } \\
\hline Allium sativum L. & Ajo & SC & Cultivada \\
\hline \multicolumn{4}{|l|}{ Anacardiaceae } \\
\hline Schinus areira L. & Molle & $\begin{array}{l}\text { ARGENTINA. Jujuy: Dpto. Humahuaca. Ocumazo, } \\
\text { 2314'19.49"S 65¹6'27.31"O., 21-II- 2021, Tortoni } 52\end{array}$ & Nativa \\
\hline \multicolumn{4}{|l|}{ Apiaceae } \\
\hline Azorella compacta Phil. & Yareta & SC & Nativa \\
\hline Azorella sp. & $\begin{array}{l}\text { Espina } \\
\text { amarilla }\end{array}$ & M-GEMA85 & Nativa \\
\hline \multicolumn{4}{|l|}{ Asteraceae } \\
\hline $\begin{array}{l}\text { Baccharis boliviensis } \\
\text { (Wedd.) Cabrera }\end{array}$ & Tolilla & $\begin{array}{l}\text { ARGENTINA. Jujuy: Dpto. Humahuaca. Ocumazo, } \\
23^{\circ} 14^{\prime} 19.49^{\prime \prime} \text { S 65'16'27.31"O., 21-II- 2021, Tortoni } 34\end{array}$ & Nativa \\
\hline Senecio nutans Sch. Bip. & Chachacoma & SC & Nativa \\
\hline $\begin{array}{l}\text { Xenophyllum poposa(Phil.) } \\
\text { V.A. Funk }\end{array}$ & Pupusa & M-GEMA86 & Nativa \\
\hline \multicolumn{4}{|l|}{ Brassicaceae } \\
\hline Baccharis grisebachii Hieron. & Quinchamal & $\begin{array}{l}\text { ARGENTINA. Jujuy: Dpto. Humahuaca. Ocumazo, } \\
23^{\circ} 14^{\prime} 19.49^{\prime \prime} \text { S 65¹6'27.31"O., 21-II- 2021, Tortoni } 04\end{array}$ & Nativa \\
\hline Lepidium didymum L. & Quimpe & $\begin{array}{l}\text { ARGENTINA. Jujuy: Dpto. Humahuaca. Ocumazo, } \\
23^{\circ} 14^{\prime} 19.49^{\prime \prime} \text { S 6516'27.31"O., 21-II- 2021, Tortoni } 27\end{array}$ & Nativa \\
\hline \multicolumn{4}{|l|}{ Chenopodiaceae } \\
\hline $\begin{array}{l}\text { Dysphania ambrosioides } \\
\text { (L.) Mosyakin \& Clemants }\end{array}$ & Paico & $\begin{array}{l}\text { ARGENTINA. Jujuy: Dpto. Humahuaca. Ocumazo, } \\
23^{\circ} 14^{\prime} 19.49^{\prime \prime} S 65^{\circ} 16^{\prime} 27.31^{\prime \prime O} \text {., 21-II- 2021, Tortoni } 24\end{array}$ & Nativa \\
\hline $\begin{array}{l}\text { Dysphania mandonii (S. } \\
\text { Watson) Mosyakin \& Clemants }\end{array}$ & Arca & $\begin{array}{l}\text { ARGENTINA. Jujuy: Dpto. Humahuaca. Ocumazo, } \\
23^{\circ} 14^{\prime} 19.49 " S 65^{\circ} 16^{\prime} 27.31^{\prime \prime O} \text {., 21-II- 2021, Tortoni } 06\end{array}$ & Nativa \\
\hline \multicolumn{4}{|l|}{ Erythroxylaceae } \\
\hline Erythroxylum coca Lam. & Coca & SC & Cultivada \\
\hline \multicolumn{4}{|l|}{ Euphorbiaceae } \\
\hline Manihot esculenta Crantz & Mandioca & SC & Adventicia \\
\hline \multicolumn{4}{|l|}{ Krameriaceae } \\
\hline $\begin{array}{l}\text { Krameria lappacea (Dombey) } \\
\text { Burdet \& B.B. Simpson }\end{array}$ & Chipichape & $\begin{array}{l}\text { ARGENTINA. Jujuy: Dpto. Humahuaca. Ocumazo, } \\
23^{\circ} 14^{\prime} 19.49^{\prime \prime} \text { 6 } 65^{\circ} 16^{\prime} 27.31^{\prime \prime O} \text {., 21-II- 2021, Tortoni } 43\end{array}$ & Nativa \\
\hline \multicolumn{4}{|l|}{ Lamiaceae } \\
\hline Rosmarinus officinalis L. & Romero & SC & Cultivada \\
\hline $\begin{array}{l}\text { Clinopodium gilliesii } \\
\text { (Benth.) Kuntze }\end{array}$ & Muña muña & $\begin{array}{l}\text { ARGENTINA. Jujuy: Dpto. Humahuaca. Ocumazo, } \\
\text { 2314'19.49"S 65¹6'27.31"O., 21-II- 2021, Tortoni } 44\end{array}$ & Nativa \\
\hline \multicolumn{4}{|l|}{ Lauraceae } \\
\hline $\begin{array}{l}\text { Cinnamomum } \\
\text { camphora (L.) J.Presl }\end{array}$ & Alcanfor & SC & Cultivada \\
\hline \multicolumn{4}{|l|}{ Loranthaceae } \\
\hline $\begin{array}{l}\text { Ligaria cuneifolia (Ruiz } \\
\& \text { Pav.) Tiegh. }\end{array}$ & $\begin{array}{l}\text { Solda que } \\
\text { solda }\end{array}$ & $\begin{array}{l}\text { ARGENTINA. Jujuy: Dpto. Humahuaca. Ocumazo, } \\
23^{\circ} 14^{\prime} 19.49^{\prime \prime} S 65^{\circ} 16^{\prime} 27.31 " O ., 21-I I-2021 \text {, Tortoni } 46\end{array}$ & Nativa \\
\hline \multicolumn{4}{|l|}{ Plantaginaceae } \\
\hline Plantago major L. & $\begin{array}{l}\text { Llantén } \\
\text { blanco, } \\
\text { llantén }\end{array}$ & $\begin{array}{l}\text { ARGENTINA. Jujuy: Dpto. Humahuaca. Ocumazo, } \\
\text { 2314'19.49"S 65¹6'27.31"O., 21-II- 2021, Tortoni } 19\end{array}$ & Adventicia \\
\hline
\end{tabular}




\section{G. L. Tortoni et al. - Preparaciones medicinales de Ocumazo}

\begin{tabular}{|c|c|c|c|}
\hline Familia/ nombre científico & $\begin{array}{l}\text { Nombre } \\
\text { popular }\end{array}$ & Voucher, fecha y lugar & Estatus \\
\hline \multicolumn{4}{|l|}{ Pteridaceae } \\
\hline $\begin{array}{l}\text { Argyrochosma nivea } \\
\text { (Poir.) Windham }\end{array}$ & Tupisaire & $\begin{array}{l}\text { ARGENTINA. Jujuy: Dpto. Humahuaca. Ocumazo, } \\
23^{\circ} 14^{\prime} 19.49^{\prime \prime} \text { 6 6516'27.31"O., 21-II- 2021, Tortoni } 47\end{array}$ & Nativa \\
\hline \multicolumn{4}{|l|}{ Rutaceae } \\
\hline Citrus limon (L.) Osbeck & Limón & SC & Cultivada \\
\hline Citrus sp. & $\begin{array}{l}\text { Naranja } \\
\text { monteña }\end{array}$ & SC & Cultivada \\
\hline \multicolumn{4}{|l|}{ Scrophulariaceae } \\
\hline Buddleja tucumanensis Griseb. & $\begin{array}{l}\text { San Juan } \\
\text { cora }\end{array}$ & $\begin{array}{l}\text { ARGENTINA. Jujuy: Dpto. Humahuaca. Ocumazo, } \\
23^{\circ} 14^{\prime} 19.49^{\prime \prime} \text { 6 65'16'27.31"O., 21-II- 2021, Tortoni } 05\end{array}$ & Nativa \\
\hline \multicolumn{4}{|l|}{ Solanaceae } \\
\hline Nicotiana glauca Graham & Cacala & $\begin{array}{l}\text { ARGENTINA. Jujuy: Dpto. Humahuaca. Ocumazo, } \\
23^{\circ} 14^{\prime} 19.49^{\prime \prime} \text { 6 6516'27.31"O., 21-II- 2021, Tortoni } 41\end{array}$ & Nativa \\
\hline $\begin{array}{l}\text { Solanum argentinum } \\
\text { Bitter \& Lillo }\end{array}$ & Hediondilla & SC & Nativa \\
\hline \multicolumn{4}{|l|}{ Verbenaceae } \\
\hline Verbena sp. & Verbena & $\begin{array}{l}\text { ARGENTINA. Jujuy: Dpto. Humahuaca. Ocumazo, } \\
23^{\circ} 14^{\prime} 19.49^{\prime \prime} \text { 65¹6'27.31"O., 21-II- 2021, Tortoni } 36\end{array}$ & Nativa \\
\hline
\end{tabular}

de una "pomada" compuesta de especies vegetales molidas con piedra (cutana), posteriormente cernidas y combinadas con unto -nombre que dan los pobladores a la grasa que recubre las vísceras de cerdo (Sus Scrofa domesticus), específicamente al riñon y/o tustuca - grasa del pecho - de la llama (Lama glama). Adicionalmente, se relevaron conocimientos de la asociación de unto con las especies Buddleja tucumanensis, Nicotiana glauca y Plantago major para el tratamiento de quemaduras. Las hojas de dichas especies son utilizadas -de a una especie por vez- para vehiculizar el unto, confeccionando de ese modo un "parche" que tendría la función de sacar el calor de la herida.

Para el tratamiento de heridas o quemaduras se cita la propiedad antibiótica de una preparación (Tabla 2, $\mathrm{N}^{\circ} 4$ ) aplicada mediante el lavado de las lesiones.

En relación al contexto de uso conviene destacar que, si bien la receta $\mathrm{N}^{\circ} 3$ (Tabla 2) es detallada con el stock de especies indicado, frente a la situación de encontrarse en centros urbanos y no disponer de Baccharis grisebachii, una especie nativa abundante en el campo, se procede a la preparación de la infusión omitiendo este componente o remplazándolo por Rosmarinus officinalis, especie cultivada de fácil acceso tanto en áreas rurales como urbanas.

\section{Preparaciones de uso interno}

Las infusiones (Tabla 2, $\mathrm{N}^{\circ} 1,8,9$ y 10) se realizan vertiendo el agua caliente sobre el producto vegetal, indicación que ha sido especialmente expresada por las y los entrevistados, ya que advierten que "No se hierve el yuyo...hay que echarle el agua hervida y dejar reposar 5 o 10 minutos, sino se pierde todo", refiriendo de esta forma a que la decocción no sería el procedimiento adecuado para mantener las propiedades medicinales de las plantas. Luego, el consumo de la infusión puede realizarse a altas temperaturas, adicionando miel o cera de abejas, o a temperatura ambiente, incluso frías denominadas como agua de, por ejemplo "agua de llantén y hediondilla" (Tabla 2, $\left.\mathrm{N}^{\circ} 11\right)$. Esta expresión refiere a su vez a un proceso que las personas detallan frente al interrogante de cómo elaboran el agua de determinada especie, o conjunto de especies, proceso que se adscribe como "por agua". Estas bebidas suelen prepararse en recipientes de un litro o más, ya que se beben durante el día -o varios días dependiendo del tratamiento- cada vez que la persona tiene sed. De consumo similar, la bebida $\mathrm{N}^{\circ} 7$ (Tabla 2) se obtiene a partir de una infusión de hierbas a la que se incorpora almidón de mandioca -adquirido en puestos comerciales- con el fin de espesar el líquido, y cáscara de naranja como saborizante.

Otro procedimiento, caracterizado para el preparado $\mathrm{N}^{\circ} 12$ (Tabla 2), consiste en "hacer 
Bol. Soc. Argent. Bot. 56 (3) 2021

Tabla 2. Preparaciones a base de plantas medicinales combinadas: composición (nombre científico y popular), uso y forma de administración.

\begin{tabular}{|c|c|c|c|c|}
\hline Preparaciones & Especies & Nombre popular & Uso & Forma de administración \\
\hline \multirow[t]{5}{*}{1} & Azorella sp. & Espina amarilla & $\mathrm{T}, \mathrm{GI}, \mathrm{R}$ & Baño, infusión, yerbeado \\
\hline & Citrus limon & Limón & & \\
\hline & Citrus sp. & Naranja monteña & & \\
\hline & Senecio nutans & Chachacoma & & \\
\hline & Xenophyllum poposa & Pupusa & & \\
\hline \multirow[t]{3}{*}{2} & Plantago major & Llantén, llantén blanco & $\mathrm{T}$ & Baño \\
\hline & Senecio nutans & Chachacoma & & \\
\hline & Xenophyllum poposa & Pupusa & & \\
\hline \multirow[t]{3}{*}{3} & Baccharis grisebachii & Quinchamal & $\mathrm{T}, \mathrm{OA}$ & Baño \\
\hline & Buddleja tucumanensis & San Juan Cora & & \\
\hline & Schinus areira & Molle & & \\
\hline \multirow[t]{2}{*}{4} & Plantago major & Llantén, llantén blanco & $A, D$ & Lavado \\
\hline & Rosmarinus officinalis & Romero & & \\
\hline \multirow[t]{4}{*}{5} & Allium sativum & Ajo & OA & Fricción \\
\hline & Argyrochosma nivea & Tupisaire & & \\
\hline & Cinnamomum camphora & Alcanfor & & \\
\hline & Rosmarinus officinalis & Romero & & \\
\hline \multirow[t]{3}{*}{6} & Erythroxylum coca & Coca & $\mathrm{OA}$ & Fricción \\
\hline & Lepidium didymum & Quimpe & & \\
\hline & Verbena sp. & Verbena & & \\
\hline \multirow[t]{4}{*}{7} & Citrus sp. & Naranja & OA & Bebida \\
\hline & Krameria lappacea & Chipi chape & & \\
\hline & Manihot esculenta & Almidón de mandioca & & \\
\hline & Verbena sp. & Verbena & & \\
\hline \multirow[t]{3}{*}{8} & Krameria lappacea & Chipichape & OA & Infusión \\
\hline & Ligaria cuneifolia & Solda que solda & & \\
\hline & Verbena sp. & Verbena & & \\
\hline \multirow[t]{4}{*}{9} & Baccharis boliviensis & Tolilla & $\mathrm{GI}$ & Infusión \\
\hline & Baccharis grisebachii & Quinchamal & & \\
\hline & Clinopodium gilliesii & Muña muña & & \\
\hline & Xenophyllum poposa & Pupusa & & \\
\hline \multirow[t]{2}{*}{10} & Dysphania ambrosioides & Paico & $\mathrm{Gl}$ & Infusión \\
\hline & Dysphania mandonii & Arca & & \\
\hline \multirow[t]{2}{*}{11} & Plantago major & Llantén & $\mathrm{GI}$ & Agua \\
\hline & Solanum argentinum & Hediondilla & & \\
\hline \multirow[t]{2}{*}{12} & Azorella compacta & Yareta & M & Agua \\
\hline & Azorella sp. & Espina amarilla & & \\
\hline
\end{tabular}

Abreviaturas: A: antibiótico; D: afecciones dérmicas; GI: afecciones gastrointestinales; M: diabetes; OA: afecciones óseas y musculoarticulares; R: afecciones respiratorias; $\mathrm{T}$ : afecciones templarias. 


\section{G. L. Tortoni et al. - Preparaciones medicinales de Ocumazo}

serenar", o sea, dejar la infusión en un vaso tapado toda la noche al aire libre. Esto implica que en reposo la preparación recibirá el rocío o aire particular de ese momento, para ser consumida la mañana siguiente.

\section{Preparados vinculados a fechas espirituales religiosas \\ Para la ceremonia ritual challada de la} Pachamama, celebrada en el mes de agosto, se describió el consumo de infusiones particulares. Una de ellas es elaborada con plantas espinosas (cualquier especie con presencia de espinas) con el fin de "pinchar los males" para que la persona goce de buena salud durante ese año. Otra bebida habitual en este contexto es el yerbeado (Tabla $\left.2, \mathrm{~N}^{\circ} 1\right)$ una infusión de hierbas saborizada con cítricos, a la que opcionalmente se adiciona alcohol, y se consume caliente en un recipiente compartido por las personas adultas con una bombilla de mate. Junto con otras bebidas, esta preparación es ofrendada a la Pachamama volcándola al mojón durante la celebración. Además de su importancia simbólica, por estar ligada a un evento ritual, se le atribuye la propiedad de tratar malestares gastrointestinales y respiratorios.

\section{Discusión}

Las preparaciones medicinales tradicionales constituyen una parte importante en la terapéutica de las comunidades indígenas y campesinas rurales, siendo una herramienta de uso habitual para médicos rurales o herbolarios en el NOA (Hilgert \& Gil, 2006). Para el presente estudio, los resultados sugieren que las personas referenciadas como especialistas por la comunidad - dos mujeres- poseen conocimientos comparables a los de médicos rurales o curanderos, por ejemplo, recetas de cierta complejidad elaboradas a partir de elementos vegetales combinados de forma precisa (Idoyaga Molina, 1999b). Al momento, un estudio de referencia en la temática ha planteado que en una misma comunidad los especialistas utilizan diferencialmente el stock de especies medicinales, valiéndose de preparaciones más complejas que son administradas en forma variable (baños, uso de ungüentos) respecto al resto de la población, configurándose diferentes roles dentro del sistema médico tradicional (Hilgert \& Gil, 2006). Desde un análisis preliminar, la presente contribución denota una tendencia semejante, la cual podría evaluarse en futuros estudios con mayor número de colaboradores.

Similar a lo observado por Crivos et al. (2009), las especialistas de la comunidad poseen conocimientos precisos respecto de los sitios y estaciones óptimas para abastecerse de los recursos necesarios, como también sobre las características propias de las plantas de cada lugar. Aun así, las migraciones de jóvenes del campo a la ciudad modificaron las estrategias de vida de los pobladores (Fontes, 2019), impactando también en el acceso a recursos naturales por parte de los adultos mayores.

En el presente estudio el patrón de distribución de las especies según su carácter cálido o frío difiere del descripto por Idoyaga Molina (1999a), para poblaciones criollas, que asocia a los sitios de clima frío con zonas de crecimiento de plantas consideradas frescas. Según lo referenciado por pobladores de Ocumazo, lugares que pueden interpretarse como fríos (por su elevación respecto al nivel del mar, temperatura y humedad) son señalados como hábitat de especies cálidas, utilizadas en el tratamiento de afecciones causadas por el frío. Estas dolencias se "agarran" al visitar esos espacios y exponerse largo tiempo a sus condiciones climáticas. En la misma lógica, el calor se constituye como un agente etiológico de la enfermedad en los sitios de menor elevación -como son los valles- donde las personas "se calorean". Coincidiendo con la teoría cálido-frío que postula, de forma general, un principio de complementariedad terapéutica, para tratar las afecciones frías se utilizan plantas de cualidad cálida. Estas habitan en terrenos de elevación apreciable, mayor a aproximadamente $3200 \mathrm{msnm}$. En cambio, para abordar el tratamiento de afecciones cálidas se recurre al uso de plantas consideradas frescas, las cuales tienen un patrón de distribución asociado a espacios de menor elevación geográfica. En resumen, las plantas cálidas se encuentran en lugares considerados fríos, en los cuales es probable contraer afecciones "frescas". En oposición las plantas frescas habitan en sitios cálidos que provocan afecciones de la misma naturaleza. Es decir, las especies vegetales responderían a un patrón de distribución altitudinal en función de sus cualidades templarias. Es particularmente 
novedoso que la condición de las plantas -fría o cálida- esté directamente asociada a los ambientes en los que crecen. De este modo, el clima representa un factor importante en la distribución de las especies según su condición, y también al agente etiológico principal de las enfermedades templarias en Ocumazo. Esto último, difiere de bibliografía previa que señala no solo a factores climáticos como causantes de dolencias, sino también al consumo de alimentos demasiado fríos o calientes (Hilgert, 2001). Por otro lado, para el tratamiento de las afecciones en Ocumazo se observa una lógica general de complementariedad, mientras que en las Yungas es habitual la combinación de la planta de condición opuesta a la enfermedad con una especie neutra, con el fin de evitar que se genere un shock en el cuerpo (Hilgert, 2001). Sería interesante indagar al respecto en futuros estudios en Ocumazo, para profundizar el análisis.

Las nociones de calor y frío como agentes causales de enfermedades, se hicieron presentes en los relatos acerca de diferentes padecimientos. Ya sea desde la contribución de un preparado a la extracción de calor en una herida, hasta las afecciones que vinculan a los síntomas directamente con el desequilibrio templario, como es el caso del refrío. En esta línea, los conceptos cálido y frío se establecen desde diferencias de temperatura y los tratamientos se enmarcan en la lógica de los modelos de regulación (Idoyaga Molina 2002 cp Martínez \& Planchuelo, 2003) los cuales basan la terapéutica en la administración de medicinas con cualidades térmicas complementarias a las de la enfermedad. En coincidencia con otras publicaciones (Idoyaga Molina \& Sarudiansky 2011; Montani, 2012), las afecciones óseas músculo-articulares (OA) son consideradas una enfermedad fría. De este modo, para el caso de estudio, los síntomas asociados a dolencias templarias circunscriben principalmente a malestares en el sistema óseo y músculoarticular. En otros sitios de estudio en el NOA, el resfrio refiere a un enfriamiento en el sistema digestivo y respiratorio (Hilgert, 2001), mientras que en Ocumazo el mismo término se utiliza para describir sintomatologías asociadas a afecciones óseas músculo-articulares.

La prevalencia del uso de plantas medicinales nativas ha sido relevada en estudios etnobotánicos anteriores, en comunidades indígenas de otros puntos de Argentina, entre ellas guaraníes
(Kujawska et al. 2017), mapuches (Molares \& Ladio, 2009), chorotes (Scarpa, 2009) descendientes Huarpes (Montani, 2012), quechuas y aymaras del NOA (Hilgert, 2001; Hilgert \& Gil, 2006). El presente trabajo constituye el primer reporte de que esta generalidad también es propia de la comunidad indígena Omaguaca.

La composición de las preparaciones respecto a las especies, al menos para el caso detallado, parece no ser estricta sino variable en función de la disponibilidad del recurso, pudiéndose remplazar u omitir alguno de los componentes. Este fenómeno se vincula a la idea de que el traslado de las personas desde ambientes rurales a centros urbanos provoca inexorablemente el traslado del conocimiento botánico que en ocasiones resulta modificado, transformando los usos tradicionales o revalorizándolos dentro del nuevo contexto urbano (Arenas et al. 2011). En este sentido, resta indagar acerca de los múltiples factores intervinientes en la sustitución y selección de nuevos recursos, que en comunidades atravesadas por migraciones involucran caracteres taxonómicos, morfológicos, químicos y sensoriales, entre otros (Medeiros et al. 2012). Para el caso ejemplificado, en los resultados del actual estudio, el remplazo de una especie nativa (B. grisebachii) por una cultivada (R. officinalis) podría vincularse a una similitud entre la forma de las hojas y el fuerte olor.

La información relevada acerca de la inclusión de productos animales coincide y complementa los usos citados en publicaciones previas en el NOA (Idoyaga Molina, 2001; Barbarán, 2004; Crivos et al. 2009; Idoyaga Molina \& Sarudiansky, 2011) y de otras regiones del país (Idoyaga Molina, 1999b; Scarpa, 2000; Arias Toledo et al. 2007). En particular, la confección de pomadas a partir de grasas requiere una elaboración más compleja, considerando diferentes etapas desde la obtención del recurso hasta su procesamiento, junto al material vegetal seleccionado especialmente. No obstante, las fórmulas medicinales incorporan ingredientes animales no solo como vehículo del material vegetal, sino también como el principal producto terapéutico. A saber, en los parches la hoja de la planta actúa de vehículo para la aplicación del unto. De forma similar, la grasa de cerdo y el excremento de gallina son utilizados en las Yungas frente a enfermedades oculares y de la piel (Hilgert, 2001). 


\section{G. L. Tortoni et al. - Preparaciones medicinales de Ocumazo}

A diferencia de lo relevado por otros autores en el país (Scarpa, 2000; Martínez \& Pochettino, 2004; Montani, 2012), para el consumo interno la decocción no es un proceso aconsejado en la elaboración de las preparaciones. Asimismo, es considerado nocivo para el mantenimiento de las propiedades curativas de las plantas. De manera que las infusiones y la ingesta "por agua" corresponden a las formas de administración vía oral más habituales, presentándose también la incorporación de almidón, como agente espesante, en una bebida a base de hierbas.

El proceso de serenado es habitual en la medicina tradicional española y de varios países latinoamericanos (Foster, 1953; Castañeda et al. 2017; Garzón, 2019). Algunos artículos citan esta práctica en Argentina (Scarpa, 2004; Luján \& Martínez, 2019) mencionándose en Hilgert (2001) para las Yungas, sin encontrarse referencias previas en la prepuna jujeña. Consecuentemente, para una descripción más acabada de la práctica debería indagarse sobre el significado que se le asigna a la atmósfera particular de la noche, capaz de dotar de una cualidad diferencial a las infusiones terapéuticas.

Para fines del análisis, los resultados de esta investigación han sido presentados bajo la dicotomía contexto de uso cotidiano versus (vs.) contexto de uso ritual. En otras palabras, las preparaciones útiles en afecciones que se inscriben como parte de la cotidianeidad de las personas vs. las vinculadas a momentos específicos de significación ritual/religiosa. No obstante, los límites entre los diversos contextos no son excluyentes, más aún, se articulan permanentemente; lo cual se evidencia en terapéuticas corrientes que incorporan elementos de significación ritual tal como propone Arteaga (2012). Cabe la aclaración para referir a las prácticas contextualizadas en la cotidianeidad, como lo es el tratamiento de afecciones mediante hierbas que se colectan especialmente en Semana Santa, una fecha de gran significación simbólica para el catolicismo.

Asimismo, se describió como las preparaciones asociadas a la celebración de la challada cobran un particular sentido simbólico, debido al culto ancestral hacia la Pachamama en la región (Mariscotti, 1978). En este contexto ritual, se destaca el significado asignado a las especies “espinosas" empleadas en el yerbeado. Esta característica en su morfología externa, a la vez que orienta su clasificación, les otorga una propiedad preventiva y curativa, tal como propone la lógica de semejanza por analogía. Este razonamiento, proviene de la Teoría de la Signatura (siglo XVI), que postulaba que las características externas de una planta daban cuenta de los efectos curativos de la misma (Martínez \& Planchuelo, 2003). Aún más, como analiza Arias Toledo (2006) acerca de la elaboración de infusiones por parte de comunidades campesinas, resulta destacable el hecho de que el yerbeado pueda elaborarse con cualquier especie, aludiendo a que la efectividad del preparado se mantiene siempre y cuando los vegetales utilizados cumplan con una condición característica responsable de otorgar el poder curativo, en este caso la presencia de espinas. En contraste con lo observado por Hilgert $(1999,20001)$ en Yungas, en Ocumazo el yerbeado no contiene yerba mate Ilex paraguariensis A. St.-Hil., sino otras especies. Es decir, ambas áreas comparten el nombre de la bebida y existen similitudes en los usos, pero la composición de especies es diferente.

Dado que las actividades terapéuticas proporcionan un contexto adecuado para caracterizar roles y modos de organización social en una comunidad (Crivos, 2007) sería conveniente que futuras investigaciones profundicen en el campo de aprendizaje de los CBT por parte de las personas que más estrategias terapéuticas combinadas despliegan, para evaluar qué identidad o rol especifico en la organización social de la comunidad representan. Al respecto, el género de las colaboradoras especializadas constituye un punto relevante a analizar en próximas investigaciones.

\section{Conclusiones}

La comunidad de Ocumazo posee un amplio repertorio de recetas basadas en sus conocimientos botánicos tradicionales acerca de preparaciones medicinales que combinan diferentes especies vegetales y productos de origen animal. La obtención de los recursos se gestiona de diferentes maneras dependiendo de la disponibilidad del material vegetal en el territorio. Las fórmulas medicinales se utilizan para tratar afecciones óseas músculo-articulares, gastrointestinales y templarias, principalmente. Se encuentran 
vigentes nociones de cálido y frío para caracterizar a las especies, ubicándolas en diferentes ambientes naturales descriptos según su cualidad. Existe una variedad de formas de administración de los tratamientos, tanto de uso externo como interno. En contexto ritual / religioso, la comunidad de Ocumazo despliega conocimientos diferenciales a los aplicados frente a afecciones propias del marco cotidiano. Así, preparaciones particulares son elaboradas especialmente durante la celebración de la Pachamama.

El presente estudio aporta al cuerpo de investigaciones realizadas en el NOA sobre el conocimiento botánico tradicional de los recursos medicinales y el contexto en el cual las terapéuticas adquieren sentido. Esta contribución también evidencia problemáticas presentes, vinculadas a las estrategias que los pobladores de Ocumazo despliegan en diferentes espacios, ya sean rurales o urbanos, para poner en práctica sus CBT medicinales. Desde este punto, resulta relevante que futuras publicaciones analicen, desde el eje pasado/presente, las transformaciones vinculadas a la gestión y uso de los recursos vegetales medicinales por parte de la comunidad.

En definitiva, las preparaciones medicinales constituyen un buen ejemplo de la complejidad característica de los sistemas etnomédicos, al poseer profundidad histórica, relevancia actual, componentes mágicos/rituales y aspectos orgánicos, articulados integralmente.

\section{Contribución de los autores}

Todas las autoras participaron de la redacción del artículo. GLT llevó adelante el muestreo y análisis de datos. BAT participó del diseño de la investigación y supervisó la redacción del manuscrito. NDV colaboró en la identificación de las especies.

\section{Agradecimientos}

A los pobladores de Ocumazo, a quienes los conocimientos tradicionales aquí publicados le pertenecen, por acceder al desarrollo de esta investigación y a la colaboración especial de los participantes entrevistados. A las instituciones financiadoras: Consejo Nacional de Investigaciones Científicas y Tecnológicas (CONICET) y Secretaria de Ciencia y Técnica y Estudios Regionales, Universidad Nacional de Jujuy (SeCTER-UNJu) mediante el financiamiento del proyecto 08/A182 "La etnobotánica, la micrografía y la calidad de semillas de la flora y agrobiodiversidad de la región surandina". Agradecemos a la editora Dra. N. I. Hilgert y a los/as revisores/as por sus sugerencias que enriquecieron el artículo. Al Dr. J. P. Villalba Ulberich por su colaboración en la edición de las figuras.

\section{Bibliografía}

AAGESEN, L., C. A. SZUMIK, F. O. ZULOAGA \& O. MORRONE. 2009. Quantitative biogeography in the South America highlands - recognizing the Altoandina, Puna and Prepuna through the study of Poaceae. Cladistics 25: 295-310. https://doi.org/10.1111/j.1096-0031.2009.00248.x

ALEXIADES, M. 1995. Apuntes hacia una metodología para la investigación etnobotánica. VI Congreso Nacional de Botánica y I Simposio Nacional de Etnobotánica Vol. 4. Cuzco, Perú.

ARENAS, P. M., I. CRISTINA, J. P. PUENTES, F. B. COSTANTINO, J. A. HURRELL \& M. L. POCHETTINO. 2011. Adaptógenos: plantas medicinales tradicionales comercializadas como suplementos dietéticos en la conurbación Buenos Aires-La Plata (Argentina). Bonplandia 20: 251-264. https://doi.org/10.30972/bon.2021327

ARIAS TOLEDO, B. 2006. Aspectos cuantitativos, cualitativos y simbólicos de la medicina tradicional de los pobladores criollos de Cerro Colorado (Córdoba, Argentina). PINACO-Investigaciones sobre Antropología Cognitiva 4: 105-115.

ARIAS TOLEDO, B., L. GALETO \& S. COLANTONIO. 2007. Uso de plantas medicinales y alimenticias según características socioculturales en Villa Los Aromos (Córdoba, Argentina). Kurtziana 33: 79-88.

ARTEAGA, F. 2012. El proceso de iniciación al curanderismo en la Pampa (Argentina). Chungará (Arica) 44: 707-715.

https://doi.org/10.4067/S0717-73562012000400011

BARBARÁN, F. R. 2004. Usos mágicos, medicinales y rituales de la fauna en la Puna del Noroeste Argentino y Sur de Bolivia. Contribuciones al Manejo de Vida Silvestre en Latinoamérica 1: 1-26. 


\section{G. L. Tortoni et al. - Preparaciones medicinales de Ocumazo}

BERKES, F. 1993. Traditional ecological knowledge in perspective. In: INGLIS, J. T. Traditional ecological knowledge: Concepts and cases, pp. 1-9. Ottawa.

BIANCHETTI, M. C. 2016. Métodos de diagnóstico vigentes en los curanderos andinos del noreste argentino. Pueblo continente 23: 57-71.

CABRERA, A. L. 1971. Regiones fitogeográficas de la República Argentina. Bol. Soc. Argent. Bot. 14: 1-42.

CABRERA, A. L. 1976. Regiones Fitogeográficas Argentinas. En: PARODI, L. R. Enciclopedia Argentina de Agricultura y Jardinería 2: 1-85. W. F. Kugler, Buenos Aires, Argentina.

CABRERA, A. L. \& A. WILLINK. 1973. Biogeografía de América Latina. Programa Regional de Desarrollo Científico y Tecnológico. OEA. Washington, DC. Serie Biología. Monografía $\mathrm{N}^{\mathrm{o}} 13$.

CASTAÑEDA, R., H. GUTIÉRREZ, E. CARRILLO, A. SOTELO. 2017. Leguminosas (Fabaceae) silvestres de uso medicinal del distrito de Lircay, provincia de Angaraes (Huancavelica, Perú). Boletín Latinoamericano y del Caribe de Plantas Medicinales y Aromáticas 16: 136-149.

CRIVOS, M. 2007. El estudio de la narrativa de casos: una propuesta para el abordaje etnográfico de las alternativas médicas. En: IDOYAGA MOLINA, A. (ed.), Los caminos terapéuticos y los rostros de la diversidad, pp. 87-113. CAEA-IUNA, Ciudad Autónoma de Buenos Aires, Argentina.

CRIVOS, M. A., M. R. MARTÍNEZ \& M. L. POCHETTINO. 2009. Fitoterapia en los Valles Calchaquíes, Salta, Argentina. En: VIGNALE N.D. Y M. L. POCHETTINO (eds.) Avances sobre plantas medicinales andinas, pp. 45- 86. CYTED - Programa Iberoamericano Ciencia y Tecnología para el Desarrollo, San Salvador de Jujuy, Argentina.

CURRIER, R. L. 1966. The hot-cold syndrome and symbolic balance in Mexican and SpanishAmerican folck medicine. Ethnology 5: 251-263. https://doi.org/10.2307/3772771

DROVETTA, R. I. 2009. Biomedicina y políticas de salud reproductiva en un contexto rural indígena de la puna jujeña, Argentina. Barbarói 31: 139- 154.

FONTES, C. 2017. La procesión de la Virgen del Rosario de Ocumazo: memoria, movimiento e identidad en el paisaje andino. Estudios Sociales del NOA 20: 25-48.

FONTES, C. 2019. Recuerdos del movimiento. Cuerpo, paisaje y memoria en la Quebrada de Humahuaca. Mundo de Antes 13: 113-139.
FOSTER, G. M. 1953. Relationships between Spanish and Spanish-American folk medicine. The Journal of American Folklore 66: 201- 217. https://doi.org/10.2307/537230

GARCÍA MORITÁN, M. \& M. B. CRUZ. 2011. Comunidades originarias y grupos étnicos de la provincia de Jujuy. Población \& Sociedad 19: 155-173.

GARZÓN, L. P. 2019. Usos medicinales asociados a la uña de gato (Uncaria tomentosa (Willd. ex Roemer \& Schultes) DC y Uncaria guianensis (Aublet) JF Gmel) en comunidades tikuna del sur de la amazonia colombiana. Ethnoscientia 4: 1-15.

http://dx.doi.org/10.18542/ethnoscientia.v0i0.10253

GUBER, R. 2001. La observación participante. En: Grupo Editorial Norma (ed.) La etnografía, método, campo $y$ reflexividad. - Enciclopedia latinoamericana de sociocultura y comunicación, pp. 55- 74. Grupo Editorial Norma, Bogotá, Colombia.

HERNÁNDEZ SAMPIERI, R., C. FERNÁNDEZ COLLADO \& P. BAPTISTA LUCIO. 2010. Metodología de la investigación, Vol. 3. McGrawHill, México.

HILGERT, N. I. 1999. Las plantas comestibles en un sector de las Yungas meridionales (Argentina). Anales del Jardín Botánico de Madrid 57: 117-138. https://doi.org/10.3989/ajbm.1999.v57.i1.194

HILGERT, N. I. 2001. Plants used in home medicine in the Zenta River basin, Northwest Argentina. Journal of ethnopharmacology 76: 11-34. https://doi.org/10.1016/S0378-8741(01)00190-8

HILGERT, N. I. 2004. Las plantas en las festividades religiosas de la selva andina argentina. Société Suisse des Américanistes 68: 37- 49.

HILGERT, N. I. 2009. La salud en las Yungas. ¿Cuáles son los principales problemas según la medicina tradicional y la formal? En: VIGNALE, N. D. \& M. L. POCHETTINO (eds.), Avances sobre plantas medicinales andinas, pp. 1-43. CYTED Programa Iberoamericano Ciencia y Tecnología para el Desarrollo, San Salvador de Jujuy, Argentina.

HILGERT, N. I., \& G. E. GIL. 2006. Medicinal plants of the Argentine Yungas plants of the Las Yungas biosphere reserve, Northwest of Argentina, used in health care. Biodiversity \& Conservation 15: 25652594. https://doi.org/10.1007/s10531-005-3874-6

HILGERT, N. I. \& G. E. GIL. 2007. Reproductive medicine in northwest Argentina: traditional and institutional systems. Journal of Ethnobiology and Ethnomedicine 3: 19. https://doi.org/10.1186/1746-4269-3-19 
IBODA. 2019. Instituto de Botánica Darwinion. Flora del Cono Sur Catálogo de Plantas Vasculares [online]. Disponible: http://www2.darwin.edu.ar/Proyectos/ FloraArgentina [Acceso: Abril 2021]

IDOYAGA MOLINA, A. 1999a. El simbolismo de lo cálido y lo frío. Reflexiones sobre el daño, la prevención y la terapia entre los criollos de San Juan (Argentina). Mitológicas 14: 7-27.

IDOYAGA MOLINA, A. 1999b. La selección y combinación de medicinas entre la población campesina de San Juan (Argentina). Scripta Ethnologica 21: 7-33.

IDOYAGA MOLINA, A. 2001. Etiologías, síntomas y eficacia terapéutica. El proceso diagnóstico de la enfermedad en el noroeste Argentino y Cuyo. Mitológicas 16: 9-43.

IDOYAGA MOLINA, A. \& M. SARUDIANSKY. 2011. Las medicinas tradicionales en el noroeste argentino: Reflexiones sobre tradiciones académicas, saberes populares, terapias rituales y fragmentos de creencias indígenas. Argumentos 24: 315-337.

ISE (INTERNATIONAL SOCIETY OF ETHNOBIOLOGY). 2008. Code of Ethics [online]. Disponible: http://ethnobiology.net/code-of-ethics/.

KUJAWSKA, M., N. I. HILGERT, H. A. KELLER \& G. GIL. 2017. Medicinal plant diversity and intercultural interactions between indigenous guarani, criollos and polish migrants in the subtropics of Argentina. PLoS ONE 12: e0169373. https://doi.org/10.1371/journal.pone.0169373

LUJÁN, M. C. \& G. J. MARTÍNEZ. 2019. Etnobotánica médica urbana y periurbana de la ciudad de Córdoba (Argentina). Bol. Latinoam. Caribe Plant Med. Aromat. 18: 155 - 196.

MARISCOTTI, A.M. 1978. Pachamama Santa Tierra. Indiana. Berlín.

MARTÍNEZ, G. J. 2008. Traditional practices, beliefs and uses of medicinal plants in relation to maternalbaby health of Criollo woman in central Argentina. Midwifery 24: 490-502.

https://doi.org/10.1016/j.midw.2006.12.011

MARTÍNEZ, G. J. 2011. Pluralismo médico y etnomedicina entre los Tobas (Qom) del Río Bermejito (Chaco, Argentina). Desafíos y aportes para una gestión intercultural de la salud en el impenetrable chaqueño. Revista del Museo de Antropología 4: 195-210. https://doi.org/10.31048/1852.4826.v4.n1.5491

MARTÍNEZ, G. J. \& A. M. PLANCHUELO. 2003. La medicina tradicional de los criollos campesinos de
Paravachasca y Calamuchita, Córdoba (Argentina). Scripta Ethnologica 25: 83-116.

MARTÍNEZ, M. R. \& M. L. POCHETTINO. 2004. Análisis de los recursos terapéuticos utilizados en el valle Calchaquí: las mujeres dicen acerca de dolencias y "remedios". Relaciones- Sociedad Argentina de Antropología 29: 163-182.

MEDEIROS, P. M. D., G. T. SOLDATI, N. L. ALENCAR, I. VANDEBROEK, A. PIERONI, N. HANAZAKI \& U. P. ALBUQUERQUE. 2012. The use of medicinal plants by migrant people: adaptation, maintenance, and replacement. Evidence-Based Complementary and Alternative Medicine 2012: 1-12. https://doi.org/10.1155/2012/807452

MOLARES, S. \& A. LADIO. 2009. Ethnobotanical review of the Mapuche medicinal flora: use patterns on a regional scale. Journal of ethnopharmacology 122: 251-260.https://doi.org/10.1016/j.jep.2009.01.003

MONTANI, M. C. 2012. Uso medicinal de plantas entre descendientes huarpes en la comunidad de Lagunas del Rosario (Mendoza, Argentina). En: ARENAS, P. (ed.). Etnobotánica en zonas áridas y semiáridas del Cono Sur de Sudamérica, pp. 251- 270. Consejo Nacional de Investigaciones Científicas y Técnicas, Buenos Aires, Argentina.

MUÑOZ, N. G. 2014. Estudio de una iniciativa de turismo comunitario asociada a pueblos originarios en el norte argentino: la experiencia Ocumazo, Jujuy, Argentina. Repositorio Digital del Ministerio de Turismo de Argentina, pp. 19.

OMS (ORGANIZACIÓN MUNDIAL DE LA SALUD). 2003. Armonización de los sistemas de salud indigenas y el sistema de salud convencional en las Américas. Washington, DC.

OMS (ORGANIZACIÓN MUNDIAL DE LA SALUD). 2013|. Estrategia de la OMS sobre medicina tradicional 2014-2023. Organización Mundial de la Salud. https://apps.who.int/iris/handle/10665/95008

POCHETTINO, M. L. \& V. LEMA. 2008. La variable tiempo en la caracterización del conocimiento botánico tradicional. Darwiniana 46: 227-239. https://www.jstor.org/stable/23228355

QUEIROZ, M. S. 1984. Hot and cold classification in traditional Iguape medicine. Ethnology. 23: 63-72. https://doi.org/10.2307/3773394

SCARPA, G. F. 2000. Estudio etnobotánico de la subsistencia de los "Criollos" del Chaco noroccidental Argentino. Tesis Doctoral. Universidad de Buenos Aires, Argentina. http://digital.bl.fcen. uba.ar/Download/Tesis/Tesis_3315_Scarpa.pdf 


\section{G. L. Tortoni et al. - Preparaciones medicinales de Ocumazo}

SCARPA, G. F. 2002. Plantas empleadas contra trastornos digestivos en la medicina tradicional criolla del Chaco noroccidental. Dominguezia 18: 36-50.

SCARPA, G. F. 2004. El síndrome cálido-fresco en la medicina popular criolla del Chaco argentino. Disparidades. Revista de Antropología 59: 5-29. https://doi.org/10.3989/rdtp.2004.v59.i2.126

SCARPA, G. F. 2009. Etnobotánica médica de los indígenas chorote y su comparación con la de los criollos del Chaco semiárido (Argentina). Darwiniana 47: 92-107. https://doi.org/10.14522/darwiniana.2014.471.43

VIGNALE, N. D. \& M. L. POCHETTINO. 2009. La investigación etnobotánica en la prospección de plantas medicinales. En: VIGNALE, N. D. \& M. L. POCHETTINO (eds.) Avances sobre plantas medicinales andinas, pp. 7- 22. CYTED Programa Iberoamericano Ciencia y Tecnología para el Desarrollo, San Salvador de Jujuy, Argentina.

VILLALBA, M. S. \& D. A. LAMBARÉ. 2019. Las manzanas en Ocumazo (Jujuy, Argentina) como parte de la diversidad de cultivos tradicionales: usos y percepciones. Bol. Soc. Argent. Bot. 54: 431-449. http://dx.doi.org/10.31055/1851.2372.v54.n3.25368 ZULOAGA, F. O., O. MORRONE \& M. J. BELGRANO. 2017. Catálogo de Plantas Vasculares del Cono Sur. Disponible en http://www.darwin.edu.ar/Proyectos/ FloraArgentina/fa.htm [Acceso: Abril 2021] 
